\title{
Communication of sexual preferences in married couples
}

\author{
JOELLYN L. ROSS \\ University of Medicine and Dentistry of New Jersey, Stratford, New Jersey \\ RUTH E. CLIFFORD \\ Stanford University Medical Center, Stanford, California \\ and \\ RUSSELL EISENMAN \\ Temple University, Philadelphia, Pennsylvania
}

\begin{abstract}
The relationship between marital satisfaction and communication of sexual behavior preferences was investigated in a sample of 61 white, middle-class married couples. Using a pairedreports questionnaire, we examined two components of communication: agreement (the number of similar preferences of the spouses) and understanding (accurate perception of the partner's preferences). Agreement was found to be significantly related to marital happiness of the couples, whereas understanding was found to be significantly related only to wives' marital satisfaction. Wives also demonstrated greater understanding of their husbands' sexual preferences than husbands did of their wives'. Other variables-length of marriage, frequency of coitus, number of children at home, and the spouses' ages and educational levels-were not shown to be related to sexual communication or marital satisfaction.
\end{abstract}

Most research on marital sexual behavior has been descriptive of the individual and has neglected the interaction between the two people involved. Two descriptive studies in the past 25 years are known to have explored, using paired reports, the ongoing sexual interaction between individuals. In the first (Mudd, Stein, \& Mitchell, 1961), couples seeking marriage counseling were questioned about their own and their partners' sexual behavior and feelings. Only frequency of coitus was reported. The second, the work of LoPiccolo and Steger (1974), examined couples who were satisfied with their sexual behavior as well as those seeking sex therapy. Norms of spouses' perceptual accuracy of each other's sexual behavior preferences were reported.

Two studies (Martin, 1975; Wallin \& Clark, 1958) used paired reports to examine the relationship between marital satisfaction and the degree of agreement partners reported regarding their preferred frequency of coitus. Both researchers found a positive relationship between the quality of the marriage and partners' feelings that they agreed.

A difficulty with these studies is that agreement in paired reports has not been placed in a theoretical context. Laing,

The authors' addresses are: Joellyn L. Ross, University of Medicine and Dentistry of New Jersey, Department of Family Practice, 40 East Laurel Rd., Suite 106, Stratford, NJ 08084; Ruth E. Clifford, Department of Psychiatry and Behavioral Sciences, Stanford University School of Medicine, Stanford University Medical Center, Stanford, CA 94305; and Russell Eisenman, Department of Psychology, Temple University, Philadelphia, PA 19122.
Phillipson, and Lee (1966) have attempted, within a framework of interpersonal perception process, to define effective communication between two people and to operationalize their concepts using paired reports. Communication, according to their approach, is based on agreement (i.e., two people having the same opinion on an issue) and understanding (i.e., each person recognizing the other's viewpoint). Laing et al. developed the Interpersonal Perception Method (IPM) to evaluate these aspects of communication. Independent responses of the partners in a dyad are paired to disclose the degree of agreement and understanding between the two people.

In applying this approach to communication about sex for this study, we have defined effective sexual communication as agreement and understanding between the partners concerning sexual attitudes and behavior preferences. The present investigation was an attempt to replicate Wallin and Clark's (1958) and Martin's (1975) results regarding sexual communication, as defined above, in relation to marital satisfaction. In addition, several other variables were hypothesized to be related to sexual communication. Longer marriages and more frequent coitus were hypothesized to correlate positively with agreement and understanding, since they may provide more opportunity to explore sexual behaviors and share feedback. Couples with more education and younger couples should have more liberal values and, thus, communicate more about sex. The presence of children in the home may inhibit sexual communication and was hypothesized to correlate negatively with agreement and understanding. Finally, the 
effect of gender on communication was examined. Women were expected to have greater understanding of their mates' preferences than men of their wives', since the male's erection and ejaculation should be more easily recognized by the partner than the female's arousal and orgasm.

\section{METHOD}

\section{Subjects}

The sample consisted of 61 white married couples living in 24 communities in Burlington, Camden, and Gloucester counties in southern New Jersey. The first author, a member of a crisis intervention hotline, attended three training meetings for hotline workers and solicited volunteer subjects. All trainees present were told that the study concerned what makes marriage successful and was an investigation of the sexual and marital relationships of married couples. Confidentiality was assured. Of the 122 individuals who participated in the study, 30 were hotline volunteers, and 25 couples had at least one partner working on the hotline. Since about $\mathbf{1 5 0}$ hotline volunteers were approached, the refusal rate among the hotline volunteers was $78 \%$. The reader should note that these individuals may be distinctive in their helping orientation and received training that may have influenced their ability to communicate in their marriages. The other 28 couples were introduced to the first author by the hotline workers who already had participated.

Subjects lived in suburbs and worked in homes, offices, and factories. The men averaged 38 years of age $(S D=10.466)$ and the women averaged 35 years $(S D=8.900)$. The mean length of marriage was 13 years $(S D=8.848)$. Fifty couples had children; the mean number was $2.5(S D=1.265)$, and the children's ages ranged from newborn to 35 (mean $=15.1, S D=14.1)$.

\section{Questionnaire Development}

Some questions concerning specific sexual preferences were derived from Hunt (1974), Kaplan (1974), and LoPiccolo and Steger (1974), whereas others were newly developed for this survey. Each spouse completed the questionnaire, first as he/she thought the partner might respond and, second, as he/she felt. Each questionnaire consisted of demographic questions, true/false statements, and fill-in-the-blank questions regarding sexual preferences.

We stressed that the questions concerned actual behaviors rather than fantasized activities. The forms for males and females were identical except for appropriate pronoun changes and the omission for males of a question concerning clitoral stimulation of self.

The construct validity of the instrument was not assessed; however, the purpose of the study was to ask spouses the same questions and to compare their responses. As a measure of degree of matching of self and other reports, the questionnaire had face validity. To maximize content validity, a wide range of marital sexual behaviors was included. Both true/false and fill-in-the-blank questions were used, since the former was convenient for scoring and the latter made the questionnaire less impersonal, thus enhancing subjects' involvement.

In assessing the instrument's test-retest reliability, reactivity of the measure had to be considered. It seemed likely that participation in the study would alter a couple's communication about sex; however, with that caution in mind, 20 women were asked to participate in a second testing. All consented and were mailed a second copy of the questionnaire about 1 month after the initial testing. Eleven women responded. Reliability, in terms of mean percentage of agreement of responses between testings, was $87 \%(S D=6.496)$. The second questionnaire contained several questions asking whether the couple discussed their responses to the first testing. All but 2 of the respondents claimed that the questionnaire provoked only limited discussion and little or no change in communication with the spouse.

The internal consistency of the questionnaire was calculated for the items concerning preferences of self and spouse. Using the KuderRichardson-20 statistic, the reliability coefficient was .63 $(S D=3.14)$.

\section{Procedure}

All couples filled out the questionnaire in their homes. Individuals sat where they could not observe each other's answers. The survey was introduced briefly by the first author as a study of marital sexual rela- tions. It was stressed that partners were not to talk with each other while filling out the questionnaire. They were urged to fill in each answer, and to guess if necessary. The administrator then sat at a distance while the questionnaires Nere completed. No interruptions occurred during this time for any couple. Each individual handed the questionnaire to the administrator when finished.

\section{RESULTS}

In summarizing the data, a problem arose concerning the number of items left blank. Four couples had to be omitted from the sample because of high numbers of blank answers. Of the 57 couples remaining, the highest number of blanks was 11 (mean = 7). Raw scores were converted to percentages of the number of questions answered by both spouses.

Answers by all subjects to the fill-in-the-blank questions were coded, using predetermined categories, by two independent raters. Interrater reliability, in terms of percentage agreement on category assignment, was $92 \%$. Discrepancies between raters were resolved by using, alternately, each rater's judgments.

Marital satisfaction was determined from each spouse's rating of the quality of the marriage on a 5-point scale. Of wives, $79 \%$, compared to $90 \%$ of husbands, rated their marriages satisfactory or excellent rather than less satisfying. The difference between wives and husbands was significant $\left[\chi^{2}(1)=54.66, p<.01\right]$. Within couples, wives and husbands tended to report similar levels of satisfaction: the correlation between spouses was moderate and significant $(r=.34, p<.005)$.

Communication was scored as follows. Agreement was defined as the total number of a wife's and husband's same responses to the same questions, adjusted according to the number of questions answered. Understanding for each spouse was defined as the number of correct matching responses of the individual for self and of the partner's perception of that individual, similarly adjusted.

The mean agreement score was $64.37(S D=13.48)$, with 100 being the highest possible score. Wives' understanding had a mean of $72.67(S D=11.89)$, and husbands' understanding had a mean of $68.63(S D=11.79)$, with 100 the highest possible score. A composite score for understanding was derived for the couple as follows: husbands' and wives' individual scores were added together, then averaged for each couple. Agreement and couples' understanding were found to be highly correlated $(r=.69, p<.001)$.

Concerning the relationship of marital satisfaction and sexual communication, results were as follows. Agreement was correlated significantly with marital satisfaction $(r=.58, p<.001$, for wives' satisfaction; $r=.30$, $p<.001$, for husbands' satisfaction). Couples with higher composite satisfaction scores showed more agreement $[t(55)=3.30, p<.002]$. Couples' understanding was significantly related to wives' marital satisfaction $(r=.30, p<.011)$, but not to husbands' satisfaction $(r=.10, p<.22)$. Couples with higher composite satisfaction scores were were not significantly different in their levels of understanding $[t(55)=1.35, p<.18]$. 
Length of marriage was examined in relation to agreement and understanding of the couple, controlling for age and education of the spouses. Partial correlation coefficients, partialling out each spouse's age and education, failed to show a significant relationship (for agreement, $r=-.03, p<.40 ;$ for understanding, $r=-.01$, $p<.47)$.

Similarly, frequency of coitus failed to display a significant relationship with sexual communication (for agreement, $r=.11, p<.20$; for understanding, $r=.09, p<.25$ ).

The relationship of education with sexual communication was tested after partialling out the effects of age and length of marriage. The results failed to support the hypothesis $(r=.06, p<.95$, for agreement; $r=.001$, $p<.99$, for understanding).

Age also was not shown to be significantly related to sexual communication, after partialling out the effects of length of marriage $(r=-.05, p<.95$, for agreement; $r=-.11, p<.90$, for understanding).

Children below age 18 were defined as at home. Couples with any children below age 18 were compared with those with no children, and were not shown to differ significantly in their sexual communication $(F=.005$, $p<.941$, for agreement, and $F=.057, p<.945$, for understanding).

Finally, wives were shown to have greater understanding of their husbands' sexual preferences than husbands had of their wives' $[t(56)=2.08, p<.04]$. Thus, the hypothesis was supported. Within couples, wives' understanding and that of husbands' were nonsignificantly correlated, although there was some relationship $(r=.21$, $p<.06)$.

\section{DISCUSSION}

This study supported the hypothesis that wives understand their husbands' sexual preferences better than husbands understand their wives'. In addition, for wives marital satisfaction was correlated with both agreement and understanding of sexual preferences, whereas for husbands marital satisfaction was correlated with agreement but not significantly with understanding. These results suggest that the couples are carrying out traditional marital roles, as described, for example, by Parsons (1959), although not in such an extremely dichotomized manner as he suggests. The wife seemed more involved in the emotionally supportive or "expressive" aspects of the marriage than did the husband.
Although agreement and understanding about sexual behavior were significantly related to each other, the two concepts did not always relate similarly to other variables (e.g., husbands' marital satisfaction). It would seem wise for clinicians working with married couples not to assume that if a couple are in agreement about sexual preferences, they necessarily appreciate that fact, at least in sexual matters. Also, accurate perception of the partner's preferences does not necessarily reflect the partner's agreement with one's own needs. Sexual conflicts might involve either or both of these communication gaps.

Our finding that agreement is related to marital satisfaction is consistent with the results of Wallin and Clark (1958). Understanding, however, was not clearly related to satisfaction. The reader should note that in this sample, correlations may have been attenuated due to the limited range of these variables. This group was skewed in terms of generally high satisfaction with marriage and high levels of agreement and understanding. Whether these parameters are representative of the general population is unknown, but it seems likely that they are biased in a positive direction. Social desirability of responses to the questionnaire also may be implicated.

Sexual communication was not found to be related significantly to most of the background demographic variables studied. Effective sexual communication did not appear to be dependent on opportunity to interact or on sociological group membership alone. Although agreement and understanding may not be so influenced, the process by which they are reached may be. A future study might examine the means of communication in various groups, comparing, for example, the use of verbal and nonverbal cues.

\section{REFERENCES}

Hunt, M. (1974). Sexual behavior in the 1970's. Chicago: Playboy Press.

Kaplan, H. S. (1974). The new sex therapies. New York: Brunner/Mazel.

Laing, R. D., Phillipson, H., \& Lee, A. R. (1966). Interpersonal perception: $A$ theory and a method of research. London: Tavistock.

LoPiccolo, J., \& STEGER, J. C. (1974). The Sexual Interaction Inventory: A new instrument for assessment of sexual dysfunction. Archives of Sexual Behavior, 3, 585-595.

MArTin, P. A. (1975). Male-female differences in desired frequency of intercourse. Medical Aspects of Human Sexuality, 11, 108-117.

Mudd, E. H., Stein, M., \& Mitchell, H. E. (1961). Paired reports of sexual behavior of husbands and wives in conflicted marriages. Comprehensive Psychiatry, 2, 149-155.

Parsons, T. (1959). The social structure of the family. In R. N. Anshen (Ed.), The family: Its function and destiny. New York: Harper.

Waluin, P., Clark, A. L. (1958). Marital satisfaction and husbands' and wives' perception of similarity in their preferred frequency of coitus. Journal of Abnormal Psychology, 47, 370-373.

(Manuscript received for publication August 14, 1986.) 